\title{
Integration of Inertial Navigation System with EM-log Using $\mathrm{H}$-infinity Filter
}

\author{
Jaehyuck Cha ${ }^{1,2}$, Hojin $\mathrm{Ju}^{2}$, Chan Gook Park ${ }^{1,2^{*}}$, Kijeong Yoo ${ }^{3}$, and Chanju Park ${ }^{3}$ \\ ${ }^{1}$ Department of Mechanical and Aerospace Engineering, Seoul National University, 08826 Seoul, Korea \\ ${ }^{2}$ Automoation and Systems Research Institute, Seoul National University, 08826 Seoul, Korea \\ ${ }^{3}$ Agency for Defense Development, 34186 Daejeon, Korea
}

\begin{abstract}
This paper presents the integration of inertial navigation system (INS) with electromagnetic-log (EM-log) as an underwater navigation system using H-infinity filter for robustness from the uncertainty of the sea current model. In underwater environments, the electromagnetic signals are attenuated rapidly, so that the global navigation satellite system is not available in general. Thus, INS is usually chosen for underwater navigation, and other aiding sensors are also used to complement its accumulative errors, one of which is EM$\log$. Since an EM-log provides the relative velocity to seawater, the integrated navigation cannot be performed accurately unless the sea current speed is compensated properly. Generally, the INS and EM-log can be integrated using extended Kalman filter (EKF). However, EKF guarantees its performance when the stochastic properties of the system's process and measurement noises are perfectly known. In other words, in the presence of sea current modelling errors, the integration using the EKF is not expected to show good performance. On the other hand, H-infinity filter is a robust filter which can tolerate such uncertainties. In this paper, the integration of INS and EM-log using H-infinity filter is studied. The performance is compared with that of the EKF case by proper computer simulation.
\end{abstract}

\section{Introduction}

Autonomous underwater vehicles (AUVs) have been developed since 1970s, they are being operated to carry out their own missions. To achieve a successful operation, the vehicles need their position or attitude information, so called navigation solutions. Global navigation satellite system (GNSS) is one of the most popular navigation system on the ground. In water, however, the signals transmitted from the GNSS satellites are attenuated rapidly, hence, the system is seldom chosen for underwater navigation.

On the other hand, inertial navigation system (INS) is self-consistent, so that it is usually chosen for underwater navigation system. Nevertheless, it has a critical limitation of cumulatively increasing positioning error with time [1]. Therefore, in most cases, INS is integrated with other sensors or systems to overcome this advantage. Long baseline (LBL), short baseline (SBL), and ultrashort baseline (USBL) have been adopted as such systems [2]. But in order to operate those baseline systems, their own infrastructure must be already constructed. Doppler velocity $\log$ can be another choice [3-5], which provides the vehicle's velocity information the device mounted on. Owing to the depth range of the sensor, however, it is also not versatile enough.

To the point of this view, even though it is not popular as other sensors, electromagnetic log (EM-log) can appeal its advantage of self-consistency. EM-log is a sensor that provides velocity information, but unlike that of DVL, the output is relative velocity to water rather than absolute velocity, since the principle of EM-log is based on Faraday's law which states electromagnetic induction.

Vaisgant et al. [6] proposed a method verifying EM$\log$ measurements and further, if need, excluding them to improve INS/EM-log integration. They focused on the effect of the low-pass filter employed in the EM-log which is for the purpose of reducing measurement noise. The integration was conducted by means of Kalman filter, which requires accurate stochastic characteristics of process and measurement noises to be known [7]. In other words, the integration of INS and EM-log is optimal only when the stochastic dynamic model of sea current is perfectly known. Unfortunately, it is very hard to model the dynamics of sea current accurately.

In this paper, the integration of INS and EM-log using $\mathrm{H}$-infinity filter is presented. H-infinity filter, also called the minimax filter, is one of the basic robust filters which can tolerate such uncertainties, that is, the influence of uncertainty in sea current model which can be severe to performance of the EKF is reduced.

The remaining parts are organized as follows; the system model including sea current model and measurement model are presented in section 2. Then, section 3 develops H-infinity filter for INS/EM-log integration. The performances of EKF and H-infinity filter are compared via computer simulation and discussed in section 4 , and the last section concludes this paper.

* Corresponding author: chanpark@snu.ac.kr 


\section{System and measurement model}

\subsection{System model}

In this subsection, the system model including INS model and sea current model is presented.

\subsubsection{INS error model}

It is well-known that the vertical channel is very weakly coupled with the horizontal channels. Hence, we separated down-axis velocity and height states from general $15^{\text {th }}$ order INS model and constitutes $3^{\text {rd }}$ order damping loop in conjunction with depth gauge [8]. Since we used NED navigation frame, INS state vector is

$$
\mathbf{x}_{I N S}=\left[L, l, v_{N}, v_{E}, \phi, \theta, \psi, \nabla_{x}, \nabla_{y}, \nabla_{z}, \varepsilon_{x}, \varepsilon_{y}, \varepsilon_{z}\right]^{T}
$$

where $L$ and $l$ denotes latitude and longitude, $v$ is velocity along the subscripted direction, north and east, $\phi, \theta, \psi$ are Euler angles, roll, pitch, and yaw in order, and $\nabla, \varepsilon$ are accelerometer and gyro bias, respectively. We assumed that these biases are all random constant.

Eventually, we can define the corresponding system matrix INS error dynamic model $F_{I N S}$ and process noise covariance $Q_{I N S}$ which are $13 \times 13$ matrices [1].

\subsubsection{Sea current model}

As mentioned above, EM-log measures the relative velocity of the vehicle to the sea water, so that it is required to augment sea current velocities as states. They will be denoted by

$$
\mathbf{x}_{C}=\left[\begin{array}{ll}
v_{C_{N}} & v_{C_{E}}
\end{array}\right]^{T}
$$

In general, sea current is modelled by $1^{\text {st }}$ order Markovmodel [9], that is,

$$
\dot{v}_{C_{N, E}}=-\frac{1}{T_{C}} v_{C_{N, E}}+\sigma_{C} \sqrt{\frac{2}{T_{C}}} \xi
$$

where $v_{C}$ is sea current along the subscripted direction, north and east, $\sigma_{C}$ is root mean square (RMS) current speed, $T_{C}$ is the correlation interval of current speed, and $\xi$ is white noise following standard normal distribution. Hence, the system matrix $F_{C}$ and process noise covariance $Q_{C}$ for sea current can be determined.

To add a comment, $T_{C}$ is related to the tendency of the sea current, so that if $T_{C}$ is large, the speed of sea current varies slowly, and vice versa. On the other hand, $\sigma_{C}$ is related to the roughness of the sea current, so that if $\sigma_{C}$ is large, the sea current becomes more rough, and vice versa. Figure 1 shows the example of sea current created by Eq. (3).

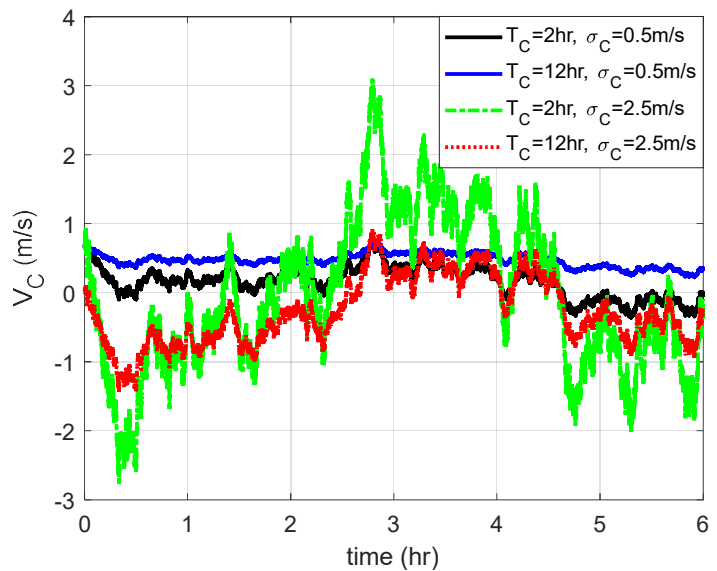

Fig. 1. Example of created sea current.

\subsubsection{Total system model}

There are no correlation between INS states and the sea current states, so that the total state vector, system matrix and process covariance matrix are difined as

$$
\begin{aligned}
& \mathbf{x}=\left[\begin{array}{ll}
\delta \mathbf{x}_{I N S}^{T} & \delta \mathbf{x}_{C}^{T}
\end{array}\right]^{T} \\
& F=\left[\begin{array}{cc}
F_{I N S} & 0_{13 \times 2} \\
0_{2 \times 13} & F_{C}
\end{array}\right] \\
& Q=\left[\begin{array}{ll}
Q_{I N S} & 0_{13 \times 2} \\
0_{2 \times 13} & Q_{C}
\end{array}\right]
\end{aligned}
$$

where $0_{m \times n}$ means zero matrix in $m \times n$ dimension.

\subsection{Measurement model}

The measurement of EM-log is not only the relative velocity of the vehicle to the water, but also that in body frame $\{B\}$. In our research, we only used longitudinal direction measurement, which is on the $\mathrm{x}$-axis of forwardright-down body frame we used. Hence, coordinate transformation matrix is used in order to mediate the state and the measurement. Therefore, considering the perturbation of attitude, the observation matrix for total states is obtained by

$$
H=\left[\begin{array}{lllll}
0_{1 \times 2} & H_{12} & H_{13} & 0_{1 \times 6} & H_{15}
\end{array}\right]
$$

where

$$
\begin{gathered}
H_{12}=\left[\begin{array}{lll}
1 & 0 & 0
\end{array}\right] \times C_{n}^{b} \times\left[\begin{array}{ll}
1 & 0 \\
0 & 1 \\
0 & 0
\end{array}\right] \\
H_{13}=-\left[\begin{array}{lll}
1 & 0 & 0
\end{array}\right] \times C_{n}^{b}\left[\left(v^{n}-v_{C}^{n}\right) \times\right] \\
H_{15}=-\left[\begin{array}{lll}
1 & 0 & 0
\end{array}\right] \times C_{n}^{b} \times\left[\begin{array}{cc}
1 & 0 \\
0 & 1 \\
0 & 0
\end{array}\right]
\end{gathered}
$$




\section{$3 \mathrm{H}$-infinity filter for INS/EM-log}

\subsection{Basic formulation of $\mathrm{H}$-infinity filter}

The formulation of H-infinity filter is summarized as [7]

$$
\begin{gathered}
\mathbf{z}_{k}=L_{k} \mathbf{x}_{k} \\
\bar{S}_{k}=L_{k}^{T} S_{k} L_{k} \\
K_{k}=P_{k}\left(I-\theta \bar{S}_{k} P_{k}+H_{k}^{T} R_{k}^{-1} H_{k} P_{k}\right)^{-1} H_{k}^{T} R_{k}^{-1} \\
\hat{\mathbf{x}}_{k+1}=F_{k} \hat{\mathbf{x}}_{k}+F_{k} K_{k}\left(\mathbf{y}_{k}-H_{k} \hat{\mathbf{x}}_{k}\right) \\
P_{k+1}=F_{k} P_{k}\left(I-\theta \bar{S}_{k} P_{k}+H_{k}^{T} R_{k}^{-1} H_{k} P_{k}\right)^{-1} F_{k}^{-1}+Q_{k}
\end{gathered}
$$

where $\mathbf{z}_{k}$ is our interest, $S_{k}$ is a weight matrix for $\mathbf{z}_{k}$, and $\theta$ is the user-specified performance bound. In addition, $\mathbf{y}_{k}$ is a measurement, and $\hat{\mathbf{a}}$ denotes the estimate of $\mathbf{a}$.

Here, we should be careful that if we set $\theta$ too large, it will broke a positive-definiteness of $P_{k+1}$ in Eq. (15), which violates the peroperty of covariance matrix.

\subsection{INS/EM-log integration}

We used Eqs. (5-10) for Eqs. (13-15). In our system and measurement models, the most uncertain parameters are related to the sea current velocities, so that we chose $L_{k}$ as

$$
L_{k}=\left[\begin{array}{ll}
0_{2 \times 13} & I_{2}
\end{array}\right]
$$

where $I_{n}$ denotes identity matrix of dimension $n \times n$. And then, we set $S_{k}$ as large as possible fixing the value of $\theta$, which means increasing the robustness of $P_{k+1}$ in Eq. (15).

\section{Simulation}

\subsection{Simulation condition}

In order to verify the performance of H-infinity filter, it is compared to the optimal and suboptimal EKF. As mentioned in the introduction, it is hard to accurately parameterize the sea current model. In this simulation we used $T_{C}=2 \mathrm{hr}$ and $\sigma_{C}=0.5 \mathrm{~m} / \mathrm{s}$ for the true sea current model, and the optimal filter was adopted with the same parameters while $\sigma_{C}=0.2 \mathrm{~m} / \mathrm{s}$ was used in the suboptimal filter. For a fair comparison, we also used $\sigma_{C}=0.2 \mathrm{~m} / \mathrm{s}$ in H-infinity filter.

The navigation trajectory was generated as shown in Figure 2, which is 24 hours long and contains stop in place motion, acceleration and deceleration, and pitching and turning maneuvers. Then, Monte-Carlo simulation with 32 runs were performed to consider random conditions.

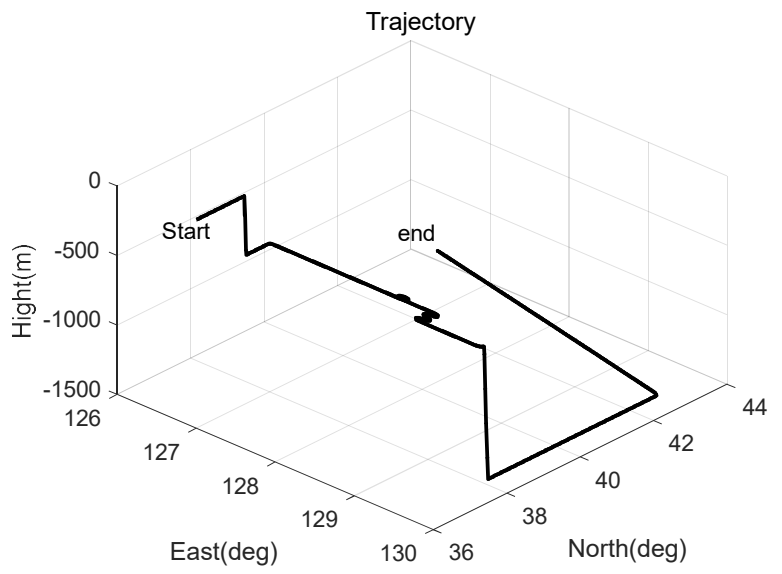

Fig. 2. 3D trajectory of simulation.

\subsection{Simulation result}

Figure 3 shows normalized latitude and longitude errors of each filter. The reason why the errors are normalized is that our interest is only in their relative magnitude. As shown in the figure, the performance of the optimal EKF is the best, which is natural result. In the suboptimal EKF case, which is conducted with inaccurate process noise model of sea current, the position estimation errors were found to increase more since 4 hours after the start of navigation. It is because using inaccurate sea current model, the filter cannot reflect its true speed, hence, the sea current estimation errors are propagated to the position estimation errors through the observation matrix. Likewise, the $\mathrm{H}$-infinity filter also performs worse in position estimation than the optimal filter. Nevertheless, it shows better results than suboptimal filter, since the designed $\mathrm{H}$-infinity filter considers the uncertainty in the stochastics of the sea current model.

Figure 4 shows further obvious difference among filter performance. The error metric presented in the figure is normalized time circular error probability (TCEP), which is defined as

$$
\operatorname{TCEP}(k)=\frac{0.589}{k} \sum_{i=1}^{k}\left[\sigma_{N}(i)+\sigma_{E}(i)\right]
$$
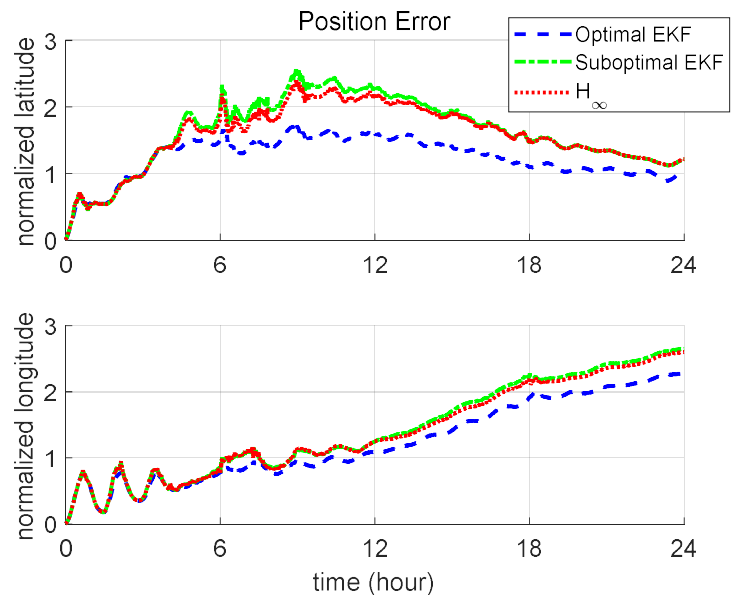

Fig. 3. Normalized latitude and longitude errors of optimal EKF, suboptimal EKF, and $\mathrm{H}$-infinity filter. 


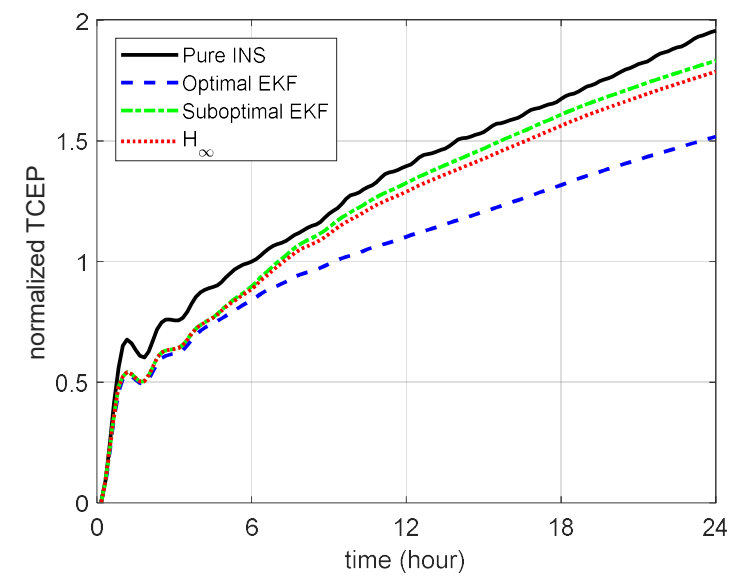

Fig. 4. Normalized time circular error probability of pure INS, optimal EKF, suboptimal EKF, and $\mathrm{H}$-infinity filter.

where $\sigma_{N}(i), \sigma_{E}(i)$ are the root mean squared error of north and east position estimates at time step $i$, respectively. Since high-grade INS has several error oscillations such as Schuler oscillation of 84.4 minutes and earth loop oscillation of 24 hours, the position errors at the final point are not always the worst. Therefore, an error metric that takes this effect into account is needed, of which TCEP is one.

The normalized TCEP and its accuracy rate of increase compared to the pure navigation of each filter at $6,12,18$, and 24 hours from the start are shown in table 1. The optimal EKF steadily improves the TCEP compared to the pure navigation over time, which is due to the fact that the biases of the inertial sensors are properly estimated by the EM-log measurement. On the other hand, those of the suboptimal filters do not so owing to the uncertain sea current model. Still, in all time domains, Hinfinity is found to outperform the suboptimal EKF.

\section{Conclusion}

In this paper, the integration of INS and EM-log using Hinfinity filter has been studied. In the presence of uncertainty in the process and measurement noise stochastics, the EKF does not guarantee the performance as desired. Since EM-log measures not the absolute velocity but the relative of the vehicle to the sea water, the estimation errors of north and east sea current velocities are augmented to the filter states. Nonetheless, the system model of the sea current states is not easy to design. That is, the inaccurate system model of the sea current degrades the performance of EKF. Therefore, $\mathrm{H}$-infinity filter, one of the most popular robust filter, has been adopted to integrate those two systems. In our research, we have designed the filter to focus on the estimation of sea current, and the required parameters are proposed in this paper. A proper computer simulation has been conducted and shown that even though the robust filter is not good as the optimal filter naturally, it outperforms the suboptimal EKF during all navigation time. The positioning errors are compared by using TCEP metric. The designed filter needs to be verified using real data as a future work.
Table 1. Normalized TCEP and accuracy rate of increase compared to the pure navigation of each filter

\begin{tabular}{|c|c|c|c|c|}
\hline Time step & Pure INS & EKF-opt & EKF-sub & H-infinity \\
\hline \multirow{2}{*}{$6 \mathrm{hr}$} & 1 & 0.8403 & 0.8968 & 0.8862 \\
\cline { 2 - 5 } & - & $15.97 \%$ & $10.32 \%$ & $11.38 \%$ \\
\hline \multirow{2}{*}{$12 \mathrm{hr}$} & 1.3950 & 1.1029 & 1.3256 & 1.2905 \\
\cline { 2 - 5 } & - & $20.94 \%$ & $4.96 \%$ & $7.5 \%$ \\
\hline \multirow{2}{*}{$18 \mathrm{hr}$} & 1.6734 & 1.3171 & 1.6094 & 1.5632 \\
\cline { 2 - 5 } & - & $21.29 \%$ & $3.82 \%$ & $6.58 \%$ \\
\hline \multirow{2}{*}{$24 \mathrm{hr}$} & 1.9561 & 1.5181 & 1.8333 & 1.7877 \\
\cline { 2 - 5 } & - & $22.40 \%$ & $6.28 \%$ & $8.61 \%$ \\
\hline
\end{tabular}

This study was supported by the research project "A Study on Multi Sensors Aided Navigation for Underwater Environment" of Agency for Defense Development.

\section{References}

1. D. Titterton, J. L. Weston, J. Weston, Strapdown inertial navigation technology (IET, 2004)

2. L. Paull, S. Saeedi, M. Seto, H. Li, IEEE J. Ocean. Eng., 39, 1 (2014)

3. D. Rudolph, T. A. Wilson, Southeastcon, 2012 Proceedings of IEEE, pp. 1-7 (2012)

4. P. A. Miller, J. A. Farrell, Y. Zhao, V. Djapic, IEEE J. Ocean. Eng. 35, 3 (2010)

5. A. Tal, I. Klein, R. Katz, Sensors, 17, 2 (2017)

6. I. B. Vaisgant, Y. A. Litvinenko, V. A. Tupysev, Gyroscopy and Navig., 2, 1 (2011)

7. D. Simon, Optimal state estimation: Kalman $H$ infinity, and nonlinear approaches (John Wiley \& Sons, 2006)

8. J. Seo, J. G. Lee, C. G. Park, $16^{\text {th }}$ IFAC Symposium on Automatic Control in Aerospace, pp. 14-18 (2004)

9. S. P. Dmitriev, V. M. Zinenko, Y. A. Litvinenko, Gyroscopy and Navig., 3, 4 (2012) 